PAPER

\title{
White matter hyperintensities are significantly associated with cortical atrophy in Alzheimer's disease
}

\author{
A A Capizzano, L Ación, T Bekinschtein, M Furman, H Gomila, A Martínez, R Mizrahi, \\ S E Starkstein
}

J Neurol Neurosurg Psychiatry 2004;75:822-827. doi: 10.1136/jnnp.2003.019273

See end of article for authors' affiliations

Correspondence to:

Dr A A Capizzano, MRI

Unit, Fernández Hospital,

Cerviño 3356 (1425),

Buenos Aires, Argentina;

andres_capizzano@

hotmail.com

Received 26 May 2003

In revised form

4 September 2003

Accepted

9 September 2003
Background and objective: Methodological variability in the assessment of white matter hyperintensities (WMH) in dementia may explain inconsistent reports of its prevalence and impact on cognition. We used a method of brain MRI segmentation for quantifying both tissue and WMH volumes in Alzheimer's disease (AD) and examined the association between $\mathrm{WMH}$ and structural and cognitive variables.

Methods: A consecutive series of 81 patients meeting NINCDS-ADRDA criteria for probable AD was studied. Nineteen healthy volunteers of comparable age served as the control group. Patients had a complete neurological and neuropsychological evaluation, and a three dimensional MRI was obtained. Images were segmented into grey matter, white matter, and cerebrospinal fluid. WMH were edited on segmented images, and lobar assignments were based on Talairach coordinates.

Results: Mild and moderate to severe AD patients had significantly more WMH than controls $(p<0.05)$. WMH preferentially involved the frontal lobes $(70 \%)$, were inversely correlated with grey matter cortical volume $\left(R^{2}=0.23, p<0.001\right)$, and were significantly associated with vascular risk factors and with a worse performance on memory tasks.

Conclusion: Objective measurements of tissue volumes in $A D$ demonstrated that WMH are significantly related to cortical atrophy and neuropsychological impairment.
W hite matter hyperintensities (WMH) is a nonspecific term that refers to white matter (WM) signal hyperintensity areas on T2 weighted MRI scans, and correlates with WM rarefaction (leucoaraiosis) as defined on CT scans. ${ }^{1}$ The main risk factors associated with development of WMH are older age and blood hypertension. ${ }^{2}$ WMH occur both in demented patients and in healthy elderly subjects, and their relevance to cognitive status has not been fully established. Healthy subjects with WMH are reported to show impairment of frontal lobe functions. ${ }^{3}{ }^{4}$ In dementia studies, some investigators found the extent of $\mathrm{WMH}$ to be significantly correlated with cognitive impairment, ${ }^{5-7}$ but others could not replicate this association. ${ }^{89}$ Furthermore, associations between WMH in dementia and depression, ${ }^{10}$ deficits in procedural memory, ${ }^{11}$ apathy, and extrapyramidal signs ${ }^{12}$ have also been reported. Discrepancies may be partly due to the variety of semi-quantitative and quantitative methods used to score $\mathrm{WMH}^{13}$ and to the heterogeneity of patients' sampling.

The anatomical basis of WMH is heterogeneous, mostly reflecting loss of myelinated axons and denudation of the ventricular ependyma. ${ }^{14}$ While periventricular WMH correspond primarily to higher fluid content, deep and subcortical WMH appear to be of vascular origin. ${ }^{15}$ Brain pathological and biochemical examination of Alzheimer's disease (AD) cases have demonstrated deep WM rarefaction with partial loss of myelin and axons. ${ }^{16}{ }^{17}$ These changes are referred to as selective incomplete WM infarction, and are thought to result from non-amyloid arteriolosclerosis associated with hypoperfusion and/or hypoxia. These findings support the suggestion that WM pathology in AD is shown on MRI scans as WMH.

The main aims of this study were to determine the severity of $\mathrm{WMH}$ at different stages of $\mathrm{AD}$, to examine $\mathrm{WMH}$ distribution by brain lobes, to assess whether WMH have a role in the process of brain atrophy in $\mathrm{AD}$, and to explore the correlation of WMH with cognitive status and neuropsychological deficits.

\section{METHODS}

Study design

Informed written consent for participation in the study was obtained from patients and caregivers, and from healthy controls. The study was approved by the institutional human subjects committee board.

\section{AD group}

This group included a consecutive series of 81 outpatients attending the Dementia Clinic of the Raúl Carrea Institute of Neurological Research for evaluation and treatment of progressive cognitive decline. The inclusion criteria were: (a) National Institute of Neurological Communicative Disorders and Stroke and Alzeimer's Disease and Related Disorders Association (NINCDS-ADRDA) criteria for probable $\mathrm{AD} ;(b)$ no history of closed head injuries with loss of consciousness, strokes, or other neurological disorder with central nervous system involvement; (c) normal results on laboratory tests (to rule out other aetiologies of dementia); and (d) no focal lesions (such as lacunes) on MRI scan. Based on the CDR and the clinical evaluation, patients were grouped into those with very mild $(C D R=0.5)$, mild $(C D R=1)$, and moderate to severe dementia $(C D R>1)$. Patients were further divided into two subgroups depending upon the volume of WMH: (a) WMH group: WMH volume $2 \mathrm{SD}$ above the mean WMH volume of the controls, and $(b)$ non-WMH group: $\mathrm{WMH}$

Abbreviations: AD, Alzheimer's disease; BNT, Boston Naming Test; CDR, Clinical Dementia Rating; COWAT, Controlled Oral Word Association Test; CSF, cerebrospinal fluid; GM, grey matter; MMSE, Mini Mental State Examination; TIV, total intracranial volume; UPDRS, Unified Parkinson's Disease Rating Scale; WM, white matter; WMH, white matter hyperintensities 

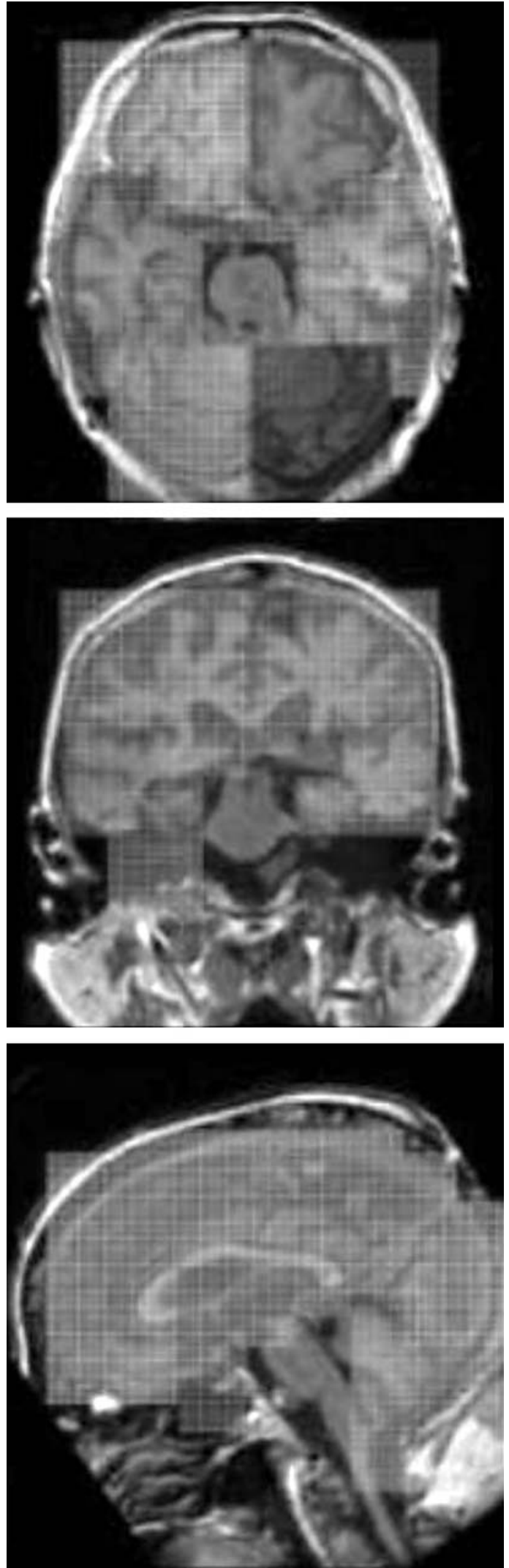

Figure 1 Automatic lobar assignments after Talairach coordinates.

volume less than 1 SD over the mean WMH control volume. Subjects with WMH volumes between 1 and 2 SD above the mean of the healthy controls $(n=13)$ were excluded from further comparisons.
Healthy control group

This group included 19 healthy elderly individuals, mostly volunteers from the community. Inclusion criteria for the group were the same as for the $\mathrm{AD}$ group, but without presence of $\mathrm{AD}$.

\section{Neurological examination}

A neurologist blind to neuroimaging findings assessed patients and controls with the following instruments: (a) Mini Mental State Examination (MMSE), ${ }^{18}$ an 11 item scale found to be valid and reliable in globally assessing a limited range of cognitive functions; $(b)$ Clinical Dementia Rating (CDR), ${ }^{19}$ a global rating device for dementia staging; and (c) Unified Parkinson's Disease Rating Scale (UPDRS), ${ }^{20}$ which assesses the presence of parkinsonian signs such as tremor, cogwheel rigidity, and bradykinesia.

\section{Neuropsychological examination}

The cognitive evaluation was carried out by a neuropsychologist blinded to the neurological and neuroimaging findings, and consisted of the following tests: (a) Token Test, ${ }^{21}$ which examines verbal comprehension of sentences of increasing complexity; (b) Boston Naming Test (BNT), ${ }^{22}$ which examines the ability to name pictured objects; (c) Controlled Oral Word Association Test (COWAT), ${ }^{23}$ which examines access to semantic information with time constraint; and (d) Buschke Selective Reminding Test, ${ }^{24}$ a measure of verbal learning and memory during a multiple trial list learning task (total recall, delayed recall, and recognition trials were used as outcome measures).

\section{Magnetic resonance imaging}

All MRI data were acquired on a 1.5 Tesla Signa Horizon system (GE, Milwaukee, USA) using a standard circularly polarised head coil. The imaging protocol included the following sequences: (a) multiplanar Flash localiser, $(b)$ coronal 3D $\mathrm{Tl}$ weighted gradient echo orthogonal to the anterior-posterior commissure (AC-PC) line (SPGR: repetition time (TR) 24; echo time (TE) $5 \mathrm{~ms}$; flip angle $40^{\circ}$; in plane resolution $0.88 \times 1.35 \mathrm{~mm}$; slice thickness $1.5 \mathrm{~mm}$; two acquisitions); and (c) coronal proton density (PD) and T2 weighted fast spin echo oriented parallel to the TI weighted images (TR $3500 \mathrm{~ms}$; TEl $32 \mathrm{~ms}$; TE2 $96 \mathrm{~ms}$; echo train length 8 ; in plane resolution $0.88 \times 1.35 \mathrm{~mm}$; slice thickness $3 \mathrm{~mm}$; no gap, one acquisition). Image post-processing was performed on a Silicon Graphics workstation (Mountain View, CA, USA) using the Brain Research: Analysis of Images, Networks and Systems software (BRAINS; Mental Health Clinical Research Center, University of Iowa Hospitals and Clinics). T1, T2, and PD images were realigned on the coronal plane orthogonal to the axial AC-PC and sagittal interhemispheric planes. Reformatted images resolution was extrapolated to $1 \mathrm{~mm}^{3}$. Images were then automatically included in a modified Talairach space, which divides the brain into 12 subregions (left and right frontal, parietal, temporal, occipital, subcortical area, and cerebellum) (fig l). The method was validated against manual lobe tracing, yielding comparable results with both techniques. ${ }^{25}$ After selection of representative pixels of grey matter (GM), WM, and cerebrospinal fluid (CSF), images were automatically segmented using a Bayesian method based upon discriminant analysis. ${ }^{26}$ Total intracranial volume (TIV) was obtained by manually editing neural net generated traces along the inner margin of the subarachnoid space. At the foramen magnum, the limit of the cranial compartment was defined by the axial plane containing the line that connected the basion with the ophistion.

WMH that appeared as hyperintense signals on PD and T2 images and as hypointense signals on $\mathrm{Tl}$ images were 

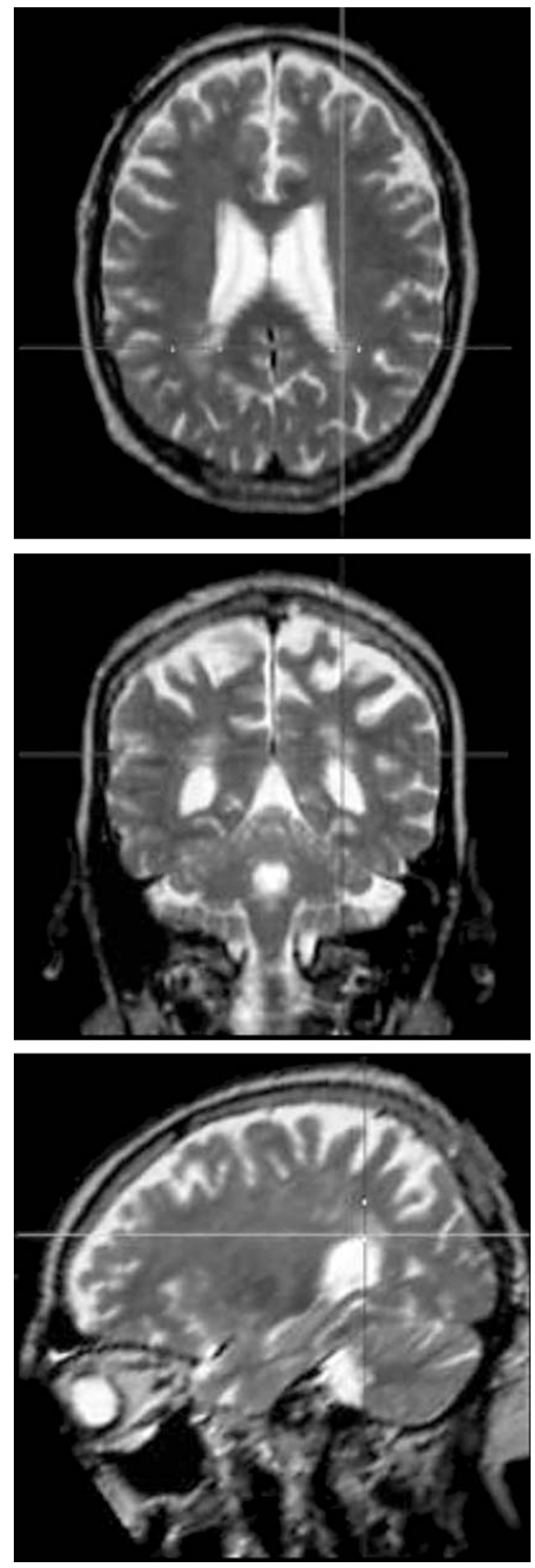

Figure 2 Tracing of WMH on T2 weighted multiplanar images using BRAINS software.

manually edited (fig 2). For this purpose, polygons surrounding the areas of WMH were traced with a mouse onto reformatted axial T2 images. Sagittal and coronal reformatted images were simultaneously evaluated for better anatomical delineation of WMH regions. BRAINS assigns WMH pixels to GM, which were reclassified after polygon tracing to the category of WMH. In order to examine the correlation between semi-quantitative and quantitative methods of $\mathrm{WMH}$ grading, a subset of consecutive images from $10 \mathrm{AD}$ patients were evaluated using the semi-quantitative WMH scoring method of Scheltens et al by a trained radiologist (AM) blinded to the quantitative MRI results. ${ }^{27}$ Lobar volumes of different tissue types (GM, WM, WMH, and CSF) were normalised to TIV and expressed as a percentage.

\section{Statistical analysis}

Statistical analysis was carried out using means, SDs, Student's $t$ tests and one way multiple analysis of covariance with age as covariable, followed by a post hoc honestly significant difference (unequal $n$ ) Tukey test. Data that were distinctly non-normal were analysed with a Kruskall-Wallis test. Outlier values for LA volume were defined as those above the 75th percentile plus 1.5 (75th-25th percentile), or below the 25 th percentile minus 1.5 ( 75 th $-25^{\text {th }}$ percentile) (Tukey). Correlations were examined with the Pearson moment correlation test and multiple regression analysis. Comparison of frequency distributions of WMH volume was performed with Fischer's exact test. All $\mathrm{p}$ values are two tailed, with significance set at 0.05 .

\section{RESULTS}

\section{Demographic findings}

$\mathrm{AD}$ patients with mild or moderate to severe dementia were significantly older than patients with very mild dementia or healthy controls $(p<0.05)$ (table 1$)$. Significant inter-group differences were not found for the remaining demographic variables. WMH volume significantly correlated with age $(\mathrm{r}=0.48, \mathrm{p}<0.001)$. The subgroup of $\mathrm{AD}$ patients with $\mathrm{WMH}$ had a significantly higher frequency of hypertension and diabetes compared with $\mathrm{AD}$ patients without $\mathrm{WMH}$ $(\mathrm{p}<0.01)$.

Correlation between quantitative and semiquantitative estimates of WMH

A subset of 10 MRIs of AD patients were evaluated with a semi-quantitative WMH scoring method by a trained radiologist (AM). Periventricular and deep WMH were scored on axially reformatted PD and T2 weighted images based on their number and size after a modification of the scoring method of Scheltens et al. ${ }^{27}$ There was a significant correlation between quantitative and semi-quantitative estimates of global WMH $(\mathrm{r}=0.94, \mathrm{p}<0.05)$ and of $\mathrm{WMH}$ of the frontal $(\mathrm{r}=0.84)$, parietal $(\mathrm{r}=0.76)$, occipital $(\mathrm{r}=0.76)$, and left temporal $(\mathrm{r}=0.83)$ lobes. Inter-rater reliability of $\mathrm{WMH}$ tracing was calculated between two trained operators in a subgroup of eight cases with varying WMH volumes. Intraclass correlation coefficients for left and right hemisphere WMH volumes were 0.97 and 0.94 , respectively.

\section{GM, WM, and WMH volumes in the different stages of $A D$}

A one way multiple analysis of covariance using the clinical category (very mild, mild, or moderate to severe $\mathrm{AD}$ and healthy controls) as the independent variable, lobar cortical GM volume (frontal, parietal, temporal, and occipital) as the dependent variables, and age as a covariable showed a significant group effect $\left(\right.$ Wilks' $\left._{(12,243)}=0.72, \quad \mathrm{p}<0.01\right)$ (table 2). Mild and moderate to severe AD patients had significantly less temporal $\left(F_{(3,95)}=9.08, \mathrm{p}<0.0001\right)$, frontal $\left(F_{(3,95)}=6.84, \mathrm{p}<0.001\right)$, and parietal $\left(F_{(3,95)}=3.62, \mathrm{p}<0.05\right)$ GM volumes compared with controls. There were no significant inter-group differences for the occipital lobe GM 
Table 1 Demographic findings

\begin{tabular}{lllll}
\hline & $\begin{array}{l}\text { Healthy } \\
\text { controls }\end{array}$ & $\begin{array}{l}\text { Very mild } \\
\text { AD }\end{array}$ & Mild AD & $\begin{array}{l}\text { Moderate to } \\
\text { severe AD }\end{array}$ \\
\hline $\begin{array}{l}\text { Number of subjects } \\
\begin{array}{l}\text { Age (years), } \\
\text { mean (SD) }\end{array}\end{array}$ & $67.7(7.8)$ & $68.2(8.3)$ & $72.8(7.7)^{*}$ & $72.7(9.4)^{*}$ \\
$\begin{array}{l}\text { Men/women } \\
\text { MMSE score, }\end{array}$ & $5 / 14$ & $13 / 14$ & $14 / 25$ & $5 / 10$ \\
mean (SD) & $28.9(1.6)$ & $26.6(2.4)$ & $20.1(3.7)$ & $13.9(3.5)$ \\
$\begin{array}{l}\text { Years of education, } \\
\text { mean (SD) }\end{array}$ & $11.5(2.7)$ & $11.1(3.6)$ & $10.2(5.4)$ & $10.2(3.1)$ \\
$\begin{array}{l}\text { Hypertension (n) } \\
\text { Diabetes (n) }\end{array}$ & 10 & 13 & 13 & 6 \\
\hline $\begin{array}{l}\text { One way analysis of variance: * } \\
\text { p }<0.05 \text { compared with controls. }\end{array}$ & 0 & 4 & 7 & 1 \\
\hline
\end{tabular}

volume. A similar one way multiple analysis of covariance with lobar WM volume as the dependent variable showed a significant group effect (Wilks' $\lambda_{(12,243)}=0.63, \mathrm{p}<0.0001$ ): all three $\mathrm{AD}$ groups had significantly lower temporal $\left(F_{(3,95)}=10.51, \quad \mathrm{p}<0.00001\right), \quad$ frontal $\quad\left(F_{(3,95)}=8.18\right.$, $\mathrm{p}<0.0001)$, parietal $\left(F_{(3,95)}=11.01, \mathrm{p}<0.00001\right)$, and occipital $\left(F_{(3,95)}=4.06, \mathrm{p}<0.01\right) \mathrm{WM}$ volumes compared with controls.

The hypothesis of unequal frequency of WMH based on the presence and severity of dementia was statistically substantiated: 13 CDR 1 patients (26\%) and 6 CDR 2-3 patients $(40 \%)$ had WMH compared with only one control $(2 \%)$ (Fischer's exact test, $\mathrm{p}<0.05$ ). No significant differences in the frequency of WMH were found between CDR 0.5 patients $(6 / 27$ patients $(22 \%)$ had $\mathrm{WMH})$ and controls $(\mathrm{p}=0.2)$. Mean (SD) \% WMH of patients and controls was distributed by brain lobes as follows: $70(21) \%$ in the frontal, $22(19) \%$ in the parietal, $3.5(5.4) \%$ in the temporal, and $1.0(2.7) \%$ in the occipital lobes (the remaining WMH volume (3.5) was located in the Talairach defined ventricular space and thus eliminated from the analysis). WMH expressed as percentage of total WM volume per lobe were as follows: frontal: 4.4 (5.6)\%, parietal: 3.8 (5.9)\%, temporal 0.6 (1.1)\%, and occipital lobe: 0.7 (1.9)\%.

\section{Correlations between brain tissue volumes}

To examine whether WMH are associated with cortical atrophy in $\mathrm{AD}$, we calculated a multiple regression analysis with total cortical volume as the dependent variable, and

\begin{tabular}{|c|c|c|c|c|}
\hline & $\begin{array}{l}\text { Healthy } \\
\text { controls }\end{array}$ & $\begin{array}{l}\text { Very mild } \\
\text { AD }\end{array}$ & Mild AD & $\begin{array}{l}\text { Moderate to } \\
\text { severe AD }\end{array}$ \\
\hline \multicolumn{5}{|c|}{ Total brain MRI segmentation } \\
\hline CGM & $41.6(2.2)$ & $41.2(2.1)$ & $39.3(30)^{*}$ & $37.2(2.5) \dagger \S$ \\
\hline WM & $32.9(3.9)$ & $30.2 i$ & 27.3 & 7)†‡ \\
\hline Total CSF & $13.2(5.5)$ & $16.5(4.1)$ & $21.5(4.7) \dagger \S$ & $24.6(3.6) \neq \S$ \\
\hline WMH & $2.7(2.4)$ & $4.6(5.9)$ & $8.0(8.5)$ & $10.1(11.2)$ \\
\hline \multicolumn{5}{|c|}{ Lobar cortical GM } \\
\hline Frontal & $16.4(1.2)$ & $16.1(1.1)$ & $15.2(1.6)^{* *}$ & $14.3(1.3) t \dagger \S \S$ \\
\hline Temporal & $10.5(0.7)$ & $10.2(0.9)$ & $9.7(0.8)^{\star *}$ & $9.1(0.7)+† \S \S$ \\
\hline Parietal & $9.5(0.6)$ & $9.5(0.7)$ & $9.1(1.0)$ & $8.6(0.7)^{* *} \ddagger \ddagger$ \\
\hline Occipital & $5.1(0.4)$ & $5.2(0.7)$ & $5.2(0.6)$ & $5.0(0.5)$ \\
\hline
\end{tabular}

All values are expressed as \% mean (SD) of TIV.

One way analysis of variance: * $\mathrm{p}<0.05$ compared with controls: $\dagger p<0.001$ compared with controls; $\ddagger p<0.01$ compared with very mild $A D ; \S p<0.001$ compared with very mild $A D$.

One way multiple analysis of covariance: for all four lobes, cortical GM, $\mathrm{p}=0.002$.

Post hoc Tukey test: ${ }^{* *} p<0.05$ compared with controls; $\uparrow+p<0.001$ compared with controls; $\ddagger \ddagger p<0.05$ compared with very mild $A D$;

$\S \S p<0.01$ compared with very mild $A D$.

CGM, cortical grey matter.
WMH volume, age, positive history of blood hypertension, and MMSE scores as the independent variables. There was a significant overall correlation (adjusted $\mathrm{R}^{2}=0.48$, $\left.F_{(2,76}=37.08, \mathrm{p}<0.01\right)$, and the most significant predictors of cortical atrophy were WMH $\left(\beta=-0.48, \mathrm{R}^{2}=0.23\right.$, $p<0.001)$, followed by MMSE scores $\left(\beta=0.36, R^{2}=0.13\right.$, $\mathrm{p}<0.001)$. WMH were significantly correlated with cortical GM volume loss in the frontal $(\mathrm{r}=-0.49, \mathrm{p}<0.001)$, temporal $(\mathrm{r}=-0.53, \mathrm{p}<0.001)$, parietal $(\mathrm{r}=-0.42$, $\mathrm{p}<0.001)$, and occipital lobes $(\mathrm{r}=-0.27, \mathrm{p}<0.01)$. In contrast, WMH were inversely correlated with WM volume in the frontal $(\mathrm{r}=-0.36, \mathrm{p}<0.001)$, and parietal lobes $(\mathrm{r}=-0.39, \mathrm{p}<0.001)$, but not in the temporal and occipital lobes (fig 3).

\section{Neuropsychological correlates of WMH}

A total of 75 individuals were assessed with the complete neuropsychological battery. A stepwise multiple regression analysis with total WMH volume as the dependent variable and the neuropsychological test scores as the independent variables showed an overall significant correlation (adjusted $\left.\mathrm{R}^{2}=0.19, F_{(6,65)}=2.56, \mathrm{p}<0.05\right)$. The only variable significantly associated with WMH was the Buschke total recall $\left(\mathrm{R}^{2}=0.14, F_{(1,70)}=11.22, \mathrm{p}<0.005\right)$.

\section{DISCUSSION}

We used a semi-automatic and fully quantified method to measure the magnitude and distribution of WMH in $\mathrm{AD}$, and there were several important findings. Firstly, AD patients with mild or moderate to severe dementia had a significantly higher frequency of WMH compared with controls. WMH mainly involved the frontal lobes $(70 \%)$ followed by the parietal lobes $(22 \%)$. In $\mathrm{AD}$ patients there was a significant association between WMH and vascular risk factors (primarily high blood pressure and diabetes) in the absence of stroke lesions on MRI. Secondly, there was a significant inverse correlation between WMH and cortical GM volume (the larger the WMH, the smaller the cortical GM volume), and this correlation was independent of age, high blood pressure, and cognitive status. Finally, there was a significant correlation between greater WMH volume and worse performance on an anterograde verbal memory task.

There are several limitations to this study. Firstly, the number of patients (primarily CDR $>1$ ) should be increased in future studies to allow the separate evaluation of patients with moderate and severe dementia. Secondly, future studies should discriminate between periventricular and deep WMH because these changes may be related to different mechanisms. Thirdly, this is a cross-sectional study that should be followed up by more appropriate longitudinal studies to determine the rate of increment of WMH across the stages of dementia. Finally, patients with 'very mild' AD may not have had probable dementia, which may explain the finding of less severe WMH than in patients with mild or moderate to severe dementia.

There are discrepant findings regarding the frequency of $\mathrm{WMH}$ in $\mathrm{AD}$ and its impact on cognitive status. Some studies using semi-quantified WMH estimation reported a higher frequency of periventricular WMH in $\mathrm{AD}$ compared with controls, ${ }^{28}$ whereas other studies reported increased WMH only in late onset $\mathrm{AD}^{27}$ and yet other investigators could not find significant differences in the magnitude of WMH between $\mathrm{AD}$ and control groups. ${ }^{29-30}$ More recent investigations using quantified segmentation techniques reported higher WMH volumes in AD $v$ controls, ${ }^{9}$ and in demented patients with $v$ those without lacunes. ${ }^{31}$ The variety of instruments used to rate $\mathrm{WMH},{ }^{13}$ different selection criteria for subjects, insufficient sample size, and heterogeneity in imaging protocols may account for these differences. In this 


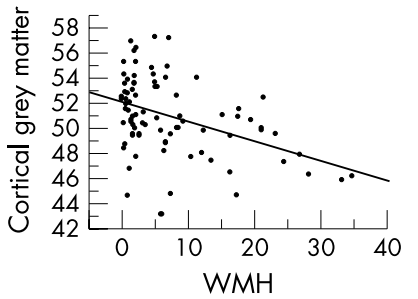

Figure 3 Significant correlation between volume of WMH and total cortical $G M$ volume for the group of $A D$ patients $(n=81$, adjusted $\left.\mathrm{R}^{2}=0.48, F_{(2,76)}=37.1, \mathrm{p}<0.01\right)$.

study we combined 3D MRI acquisition with automatic brain segmentation of GM, WM, and CSF, followed by manual tracing of $\mathrm{WMH}$, and found a significant correlation between WMH volume and semi-quantified ratings, and high interrater reliability for WMH volume calculation.

Using a quantified method, we found larger WMH volumes in $\mathrm{AD}$ patients compared with controls, with more advanced dementia showing larger WMH. The presence of lacunes or other cerebrovascular lesions has been consistently associated with more severe $\mathrm{WMH} .^{2}$ The fact that we excluded patients with lacunes may explain the lower frequency of WMH in our AD group compared with other studies. Most WMH were located in the frontal lobes, followed by the parietal lobes, and a minor proportion in temporal and occipital lobes. The difference in extent of WMH volume across lobes partially reflected different lobe sizes, with a higher proportion of WMH over total WM in the frontal and parietal lobes (mean (SD) $4.4(5.6) \%$ and 3.8 (5.9)\%, respectively) compared with temporal and occipital lobes $(0.6(1.1) \%$ and $0.7(1.9) \%$, respectively). Penetrating arteries are longest in the WM regions around the frontal horns and head of the caudate nuclei, suggesting that the higher frequency of WM pathology in the frontal lobes is due to chronic ischaemia resulting from sclerosis of penetrating arteries. ${ }^{32}$ The association of WMH with high blood pressure suggests a role for arteriolar sclerosis in the development of WMH. Furthermore, neurological and neuropsychiatric evidence of frontal lobe dysfunction was found to be significantly associated with the extent of $\mathrm{WMH}^{32-34}$ supporting the preferential vulnerability of the frontal lobes to this condition.

One of the major findings of this study was that the volume of WMH accounted for $23 \%$ of the variance of cortical GM atrophy. In other words, the more severe the WMH, the more severe the cortical atrophy. The question now arising is the direction of this correlation-that is, whether WMH may contribute to cortical atrophy in $\mathrm{AD}$, whether cortical atrophy may produce WMH, or whether both WMH and cortical atrophy are epiphenomena of another unknown factor. In a neuropathological study of $\mathrm{AD}$, Facio et $a^{35}$ reported a significant correlation between markers of cortical degeneration (senile plaques and neurofibrillary tangles) and severity of periventricular leucoencephalopathy. They proposed that cortical pathology may produce both axonal and myelin degeneration, resulting in increased water content in the periventricular WM. Recent studies proposed that smooth periventricular WMH may be related to loss of myelinated axons in the deep WM and denudation of the ependymal lining. ${ }^{14}$ On the other hand, pathological WM changes in $\mathrm{AD}$ are not topographically associated with the distribution of cortical pathology, arguing against wallerian degeneration. ${ }^{36}$

Alternatively, WMH may produce secondary atrophy in cortical regions. White matter infarction at WMH sites interrupting the axons of projecting cortical neurons may result in cortical neuronal loss by retrograde degeneration.
Disconnection between cortical and subcortical brain regions due to WMH could account for the cognitive impairment. ${ }^{37}$ $\mathrm{AD}$ patients with WMH showed reduced frontal lobe perfusion in the absence of overt cortical damage. ${ }^{33}$ Furthermore, Sultzer et $a l^{38}$ reported a significant correlation between cortical hypometabolism and the area of WMH in the anterior periventricular region, suggesting that the former may be due to deafferentation (subcorticalcortical diaschisis). Mielke et $a l^{39}$ demonstrated that the severity of dementia in patients with pure subcortical infarcts has a stronger relationship with cortical hypometabolism than with the amount of tissue destruction. In a ${ }^{1} \mathrm{H} M R$ spectroscopy study of subcortical vascular dementia, Capizzano et $a l^{40}$ reported a significant negative correlation between WMH volume and frontal cortical concentration of the neuronal marker NAA, further supporting the inverse correlation between WMH volume and cortical functioning.

Another finding of our study was that AD patients had a significantly lower volume of cortical GM compared with controls, which is in agreement with pathology and imaging data. Largest differences were found for temporal and frontal cortical volumes, with parietal lobe atrophy reaching significance only in the CDR 2-3 group compared with controls. No significant inter-group differences were found in occipital lobe volume. A significant loss of WM volume was detected in all $\mathrm{AD}$ groups compared with controls. Reduced WM volume, as measured in postmortem MRIs, was associated with dementia in the Nun Study, ${ }^{41}$ and atrophy of the corpus callosum is a well known feature of AD. ${ }^{42}$

The only significant neuropsychological correlate of WMH in this study was the Buschke total recall test, a task assessing anterograde verbal memory. A previous study of $\mathrm{WMH}$ in $\mathrm{AD}$ patients also reported impairment in verbal memory in early $\mathrm{AD}^{43}$ and the authors argued that these alterations may reflect dorsolateral prefrontal dysfunction due to cortico-subcortical disconnection. In contrast, in a previous investigation we found no significant between group differences on specific neuropsychological tasks between $\mathrm{AD}$ patients with and without WMH. ${ }^{12}$ Literature reports are divergent as to the impact of WMH upon cognitive functioning in both $\mathrm{AD}$ patients and elderly controls. In a 10 year follow up study, Swan et al ${ }^{44}$ reported that healthy subjects with larger WMH volume had greater decline on measures of planning, sequencing, response set, shifting, psychomotor speed, working memory, selective attention, and response selection compared with individuals with normal scans. Other studies also found that healthy elderly subjects with WMH had impairment on frontal lobe functions. ${ }^{3}$ Boone et al l $^{3}$ proposed that a certain "threshold" of WMH volume is required before the onset of cognitive deficits, which may explain the lack of association between WMH and cognition if most subjects are below that critical level. Furthermore, in a study of over 1000 healthy subjects, de Groot $e t a l^{45}$ found that periventricular but not subcortical WMH correlated with neuropsychological deficits (primarily psychomotor speed). Based on our finding of a significant correlation between $\mathrm{WMH}$ and cortical atrophy in $\mathrm{AD}$, the association of more severe WMH with greater deficits on a test of verbal memory could be explained by the concomitant cortical atrophy.

In conclusion, this is to our knowledge one of the first studies using a fully quantified method of brain segmentation of WMH in AD. Patients with dementia had a significantly higher volume of WMH compared with age matched healthy controls. WMH were most marked in the frontal lobes, followed by the parietal lobes, with a much smaller impact on temporal and occipital lobes. WMH accounted for $23 \%$ of the variance of cortical atrophy in $\mathrm{AD}$, 
and significantly correlated with anterograde verbal memory deficits.

\section{ACKNOWLEDGEMENTS}

We are grateful to Dr N Andreasen from the Mental Health Clinical Research Center, University of Iowa Hospitals and Clinics, for kindly providing us with the BRAINS software. We also thank Dr S Arndt from the Departments of Psychiatry and Biostatistics, University of Iowa, for his revision of the manuscript and thoughtful suggestions.

\section{Authors' affiliations}

A A Capizzano, MRI Unit, Fernández Hospital, Buenos Aires, Argentina L Ación, Department of Psychiatry, University of lowa College of Medicine, lowa City, IA, USA

T Bekinschtein, Cognitive Neurology section, Neurology Department,

"Raúl Carrea" Institute of Neurological Research, Buenos Aires,

Argentina

M Furman, Argentine Secretariat of Science and Technology, Buenos Aires, Argentina

H Gomila, Buenos Aires Neuropsychiatric Center, Buenos Aires,

Argentina

A Martínez, MRI Unit, "Raúl Carrea" Institute of Neurological Research, Buenos Aires, Argentina

R Mizrahi, Department of Psychiatry, University of Toronto, Toronto, Canada

S E Starkstein, Department of Psychiatry and Clinical Neurosciences, University of Western Australia and Fremantle Hospital, Australia

Competing interests: none declared

\section{REFERENCES}

1 Hachinsky V, Potter P, Merksey H. Leuko-Araiosis. Arch Neurol 1987;44:21-3.

2 Longstheth WT, Manolio TA, Arnold A, et al. Clinical correlates of white matter findings on cranial magnetic resonance imaging of 3301 elderly people. The Cardiovascular study. Stroke 1996;27:1274-82.

3 Boone KB, Miller BL, Lesser IM, et al. Neuropsychological correlates of whitematter lesions in healthy elderly subjects. A threshold effect. Arch Neurol 1992:49:549-54

4 De Carli C, Murphy DGM, Tranh M, et al. The effect of white matter hyperintensity volume on brain structure, cognitive performance, and cerebral metabolism of glucose in 51 healthy subjects. Neurology 1995;45:2077-84.

5 Bondareff W, Raval J, Woo B, et al. Magnetic resonance imaging and the severity of dementia in older adults. Arch Gen Psychiatry 1990:47:47-51.

6 Liu CK, Miller BL, Cummings JL, et al. A quantitative MRI study of vascular dementia. Neurology 1992;42:138-43.

7 Stout JC, Jernigan TL, Archibald SL, et al. Association of dementia severity with cortical gray matter and abnormal white matter volumes in dementia of the Alzheimer type. Arch Neurol 1996:53:742-9.

8 Giubilei F, Bastianello S, Paolillo A, et al. Quantitative magnetic resonance analysis in vascular dementia. J Neurol 1997;244:246-51.

9 Tanabe J, Amend D, Schuff N, et al. Tissue segmentation of the brain in Alzheimer's disease. AJNR Am J Neuroradiol 1997:18:115-23.

10 Barber R, Scheltens P, Gholkar A, et al. White matter lesions on magnetic resonance imaging in dementia with Lewy bodies, Alzheimer's disease, vascular dementia, and normal aging. I Neurol Neurosurg Psychiatry 1999:67:66-72.

11 Libon DJ, Bogdanoff B, Cloud BS, et al. Declarative and procedural learning, quantitative measures of the hippocampus, and subcortical white alterations in Alzheimer's disease and ischaemic vascular dementia. J Clin Exp Neuropsychol 1998;20:30-41

12 Starkstein SE, Sabe L, Vazquez S, et al. Neuropsychological, psychiatric, and cerebral perfusion correlates of leukoaraiosis in Alzheimer's disease. J Neurol Neurosurg Psychiatry 1997;63:66-72.

13 Scheltens $\mathbf{P}$, Erkinjunti T, Leys $\mathrm{D}$, et al. White matter changes on $\mathrm{CT}$ and MRI: An overview of visual rating scales. Eur Neurol 1998;39:80-9.

14 Scheltens Ph, Barkhof F, Leys D, et al. Histopathologic correlates of white matter changes on MRI in Alzheimer's disease and normal aging. Neurology 1995;45:883-8.

15 Fazekas $\mathrm{F}$, Kleinert $\mathrm{R}$, Offenbacher $\mathrm{H}$, et al. Pathologic correlates of incidental MRI white matter signal hyperintensities. Neurology 1993;43:1683-9.

16 Brun A, Englund E. Regional pattern of degeneration in Alzheimer's disease: neuronal loss and histopathological grading. Histopathology 1981;5:549-64.
17 Englund E, Brun A, Alling C. White matter changes in dementia of Alzheimer's type. Biochemical and neuropathological correlates. Brain 1988;111:1425-1439.

18 Folstein MF, Folstein SE, McHugh PR. "Mini mental state": a practical method for grading the cognitive state of patients for the clinician. J Psychiatr Res 1975; 12:189-98.

19 Hughes CP, Berg L, Danziger W, et al. A new clinical scale for the staging of dementia. Br J Psychiatry 1982;140:566-72.

20 Fahn S, Elton E. UPDRS Development Committee. Unified Parkinson's disease rating scale. In: Fahn S, Mardsen CD, Goldstein M, Calne CD, eds. Recent developments in Parkinson's disease. Florham Park, NJ, Macmillan, 1987:153-63.

21 De Renzi E, Faglioni P. Development of a shortened version of the token test. Cortex 1978;14:41-9.

22 Kaplan EF, Goodglass H, Weintraub S. The Boston naming test. Philadelphia: Lea and Febiger, 1983.

23 Lezak MD. Neuropsychological assessment, 2nd edn. New York: Oxford University Press 1983.

24 Bushke H, Fuld PA. Evaluating storage, retention, and retrieval in disordered memory and learning. Neurology 1974;24:1019-25.

25 Andreasen NC, Rajarethinam R, Cizadlo T, et al. Automatic atlas-based volume estimation of human brain regions from MR images. J Comput Assist Tomogr 1996;20:98-106.

26 Harris G, Andreasen NC, Cizadlo T, et al. Improving tissue classification in MRI: A three-dimensional multispectral discriminant analysis method with automated training class selection. J Comput Assist Tomogr 1999;23:144-54.

27 Scheltens Ph, Barkhof F, Valk J, et al. White matter lesions on magnetic resonance imaging in clinically diagnosed Alzheimer's disease: Evidence for heterogeneity. Brain 1992;115:735-48.

28 Fazekas F, Chawluk JB, Alavi A, et al. MR signal abnormalities at $1.5 \mathrm{~T}$ in Alzheimer's dementia and normal aging. AJNRAm J Neuroradiol 1987;8:421-6.

29 Leys D, Soetaert G, Petit H, et al. Periventricular and white matter magnetic resonance imaging hyperintensities do not differ between Alzheimer's disease and normal aging. Arch Neurol 1990:47:524-7.

30 Miller DS, Kumar A, Yousem DM, et al. MRI high-intensity signals in late-life depression and Alzheimer's disease. Am J Geriatr Psychiatry 1994;2:332-7.

31 Fein G, Di Sclafani V, Tanabe J, et al. Hippocampal and cortical atrophy predict dementia in subcortical ischemic vascular disease. Neurology 2000;55: 1626-35

32 Ishii N, Nishihara Y, Imamura T. Why do frontal lobe symptoms predominate in vascular dementia with lacunes? Neurology 1986;36:340-5.

33 Starkstein SE, Sabe L, Vazquez S, et al. Neuropsychological, psychiatric, and cerebral blood flow findings in vascular dementia and Alzheimer's disease. Stroke 1996;27:408-14.

34 Yamauchi $\mathrm{H}$, Fukuyama $\mathrm{H}$, Shio $\mathrm{H}$. Corpus callosum atrophy in patients with leukoaraiosis may indicate global cognitive impairment. Stroke 2000;31:1515-20.

35 Facio EJ, Vega MG, Saggese JA. Estudio anatomopatológico de la sustancia blanca periventricular en condiciones normales y patológicas. In: Enrique José Facio. Neuropatología. Recopilación de su obra publicada. Buenos Aires: Asociación de Profesionales de Salud Mental del Hospital JT Borda, 1998.

36 Brun A. Englund E. A white matter disorder in dementia of the Alzheimer type: A pathoanatomical study, Ann Neurol 1986;19:253-62.

37 Inzitari D. Age related white matter changes and cognitive impairment. Ann Neurol 2000;47:141-3.

38 Sultzer DL, Mahler ME, Cummings JL, et al. Cortical abnormalities associated with subcortical lesions in vascular dementia. Clinical and positron emission tomography findings. Arch Neurol 1995;52:773-80.

39 Mielke R, Herholz K, Grond M, et al. Severity of vascular dementia is related to volume of metabolically impaired tissue. Arch Neurol 1992;49:909-13.

40 Capizzano A, Schuff N, Amend D, et al. Subcortical ischemic vascular dementia: Assessment with quantitative MR imaging and ${ }^{1} \mathrm{H} M R$ spectroscopy. AJNR Am J Neuroradiol 2000;21:621-30.

41 Smith CD, Snowdon DA, Wang $H$, et al. White matter volumes and periventricular white matter hyperintensities in aging and dementia. Neurology 2000;54:838-42.

42 Hampel H, Teipel SJ, Alexander GE, et al. Corpus callosum atrophy is a possible indicator of region and cell type specific neuronal degeneration in Alzheimer disease. Arch Neurol 1998:55:193-8.

43 Tsiskaridze A, Shakarishvili R, Janelidze M, et al. Cognitive correlates of leukoaraiosis in the early stages of Alzheimer's disease. Funct Neurol 1998:13:17-25.

44 Swan GE, DeCarli C, Miller BL, et al. Biobehavioral characteristics of nondemented older adults with subclinical brain atrophy. Neurology 2000;54:2108-14.

45 de Groot JC, de Leeuw FE, Oudkerk M et al. Cerebral white matter lesions and cognitive function: the Rotterdam Scan Study. Ann Neurol 2000;47:145-51. 